between what is called living and dead matter may not exist, as has been suggested by Boycott.

Of the functions usually postulated for a living organism, assimilation appears to be the most characteristic and indispensable. It is reasonable to assume that the metabolism of an organism would be much simplified if it existed in a circulating medium which provided a constantly changing supply of materials resembling its own components, such as might be afforded for an obligatory parasite living inside the cells of its host. Such an existence would have very different requirements from a truly independent life.

Virus diseases are transmitted from one animal to another by very varied means. Some, like canine distemper and certain influenza-like diseases of man, by droplets in the breath, others like yellow and dengue fevers by the bites of insects, others like louping-ill of sheep by the bites of blood-sucking ticks or of mites; again, the bite of the mammalian host is the usual mode of infection with rabies, but for many others the method of transmission is still uncertain. In these respects they do not differ from diseases due to bacteria.

It is characteristic of many diseases that, although the initial infection is caused by a virus, many of the symptoms and complications are due to secondary infections with bacteria, and this is notably the case in the influenza-like group in man, in swine fever, canine distemper and swine influenza.

A virus may become remarkably adapted and sometimes permanently attenuated, when transferred to a new host, as is well instanced in the change of the virus of small-pox to vaccinia in cattle and rabbits, and of the rabies virus in the rabbit.

The period of resistance shown by the host following an attack of disease is sometimes very prolonged, even lifelong, after small-pox, varicella, yellow fever and canine distemper, but in some other cases the protection afforded is of comparatively short duration, in foot-and-mouth disease usually for one to two years, whereas frequently recurring attacks due to the virus of Herpes labialis are common.
This immunity is to a great extent due to the production in the animal body of 'antibodies' which can be found in the blood serum of recovered animals, just as occurs after bacterial infection. These antibodies can often be demonstrated by the formation of a precipitate or by the agglutination of the virus particles when a suspension of the elementary bodies is mixed with the serum, or by the neutralisation of the virus by the serum when both are injected into an animal. These phenomena are of the same kind as the precipita tion occurring when the blood serum of an animal which has been inoculated with a foreign protein (antigen) is mixed with the same protein in vitro, and are not peculiar to true infections.

It is not intended here to do more than refer to the enormous and increasing number of filterable viruses known to cause infective disease in plants and found in their juices. These, like mosaic disease of tobacco, spotted wilt of tomato, and crinkle and leaf-roll of potato, may cause very serious disease, or in other cases may be present throughout the plant without producing any visible effect, as in some infections of the potato.

There is good evidence that two viruses may co-exist in the same plant, and as a result the symptoms may be either much more or much less severe than when either virus is present alone.

Some viruses are transmitted by insects such as aphis or thrips, while others pass by unknown means. In some of these diseases of plants peculiar 'inclusion bodies' are found in certain cells, but their relation to the virus is undetermined. It is known that these 'bodies', as well as some of the symptoms due to a virus, can in special cases be imitated by the addition of certain inorganic salts to the soil, but the disease is not then transmissible. Some plant viruses are highly resistant to drying, chemical action and alcohol, and in many ways the viruses of plants resemble those of animals.

The problems which the behaviour and properties of viruses raise are of great practical and theoretical interest, and are by no means yet solved.

\title{
Heat Production of Muscle and Nerve
}

\section{By Prof. A. V. HiLl, o.B.E., F.R.s., Foulerton Research Professor of the Royal Society}

$\mathrm{T}^{\mathrm{T}}$ HE first paper by the present writer on this subject was printed almost exactly twentyfive years ago in the Journal of Physiology: the coincidence reinforced the invitation of the Editor of NATURE to write an interim report.

The heat production of muscle had been investigated in the past by such scientific giants as
Helmholtz, Heidenhain, Fick and Blix: and indeed it was with Blix's apparatus, purchased by the providence of Langley and set in order by the Cambridge Instrument Company in the days of Horace Darwin and Keith Lucas, that the present experiments began. For some time, apart from Blix's work on the relation between heat 
production and muscle length, little of importance had been done on the subject; but, as Langley wrote in a letter dated November 11, 1909, "an especial problem" had been "suggested by Fletcher and Hopkins's work on the efficiency of the muscle working with and without oxygen. . . . Once started, there are plenty of further experiments to do". The indication was clear: and in 1912 it was shown that a considerable part of the heat set free by an active muscle "occurs in recovery processes-presumably in the oxidative removal of the lactic acid liberated during contraction". The recovery heat production of muscle has been a fruitful subject of investigation in the intervening years ; the most recent papers have pushed its analysis to the extreme case of a single twitch in which, in a muscle of about $0.1 \mathrm{gm} ., 0.0002$ calorie is liberated in the fifteen minutes following contraction.

The 'initial' heat production--that which occurs during actual contraction-has been extensively explored. Its investigation has led to great improvements in technique, for not only its amount but also its distribution in time relative to the various phases of mechanical activity had to be determined. The former demanded calibration of the thermo-electric apparatus, together with the muscle, in absolute units of heat: the latter, great rapidity in recording. The heat had to be related to the force developed and maintained, to the work done, to the character, the duration and the frequency of the stimulus, to the temperature and to the physico-chemical condition of the muscle. It was shown quite early (1914) that the initial heat, and its relation to force developed, are independent of the presence of oxygen; so that the chemical processes of contraction were presumably of a non-oxidative character. This fact has a fundamental bearing on the nature of muscular fatigue of the rapid 'athletic' type: as also has the behaviour of the delayed oxidative heat production on recovery from such fatigue: but that is another story.

In 1912 the first attempt was made to relate the heat associated with the process of muscular activity to the only chemical events then known to occur : the formation and subsequent removal of lactic acid and the production of carbon dioxide. It was clear at once, as has been abundantly verified since, that the lactic acid was not removed simply by oxidation-the heat was far too small. In 1913, Peters published the first direct comparison of the initial heat with the lactic acid set free, and from 1919 onwards Meyerhof and his colleagues have made full use of the relation between heat and chemical change in their exploration of the intermediate mechanism of contraction. Lactic acid is no longer the only chemical substance known to be liberated in activity: creatine-phosphoric acid breaks down, and a complex sequence of phosphate changes has been discovered. It is probable indeed that the primary energy change in muscular activity is not lactic acid formation at all, and that the latter process is really the first stage in restoration, a non-oxidative stage rather rapidly completed, and followed (if oxygen be present) by the oxidative processes of recovery referred to previously. Here again the heat has thrown light on the problem, since, even in the complete absence of oxygen, a significant amount of heat is set free in the first minutes following muscular activity.

From the general scientific point of view, apart from the details of chemical machinery, the following broad conclusions may be drawn :

(i) The muscle is not a heat engine, but a chemical engine working at practically constant temperature.

(ii) It possesses an 'accumulator' mechanism by which energy can be liberated very rapidly without oxidation, and a 'recharging mechanism' by which the status quo can be restored under the influence of, and at the expense of energy derived from, oxidation.

(iii) It is primarily a machine for developing and maintaining force, not for doing work : even if no work is done, the muscle contracting at constant length ; even if negative work is done as when a muscle is stretched during contraction (for example, in walking downstairs), considerable heat is liberated, more the greater the duration of contraction. It is true, as Fenn (1923) found, that there are significant relations between work and heat, but these do not disguise the fact that the primary relation between thermal and mechanical effects is one between heat and force developed and maintained.

(iv) The 'efficiency' of the muscle machine, considering the whole cycle of breakdown and recovery, that is (work done)/(total energy set free) could never be greater than about 50 per cent, since the recovery process frees about as much energy as the initial process, and the latter always shows a positive balance of heat, even when maximal work is done. Under actual working conditions in man the maximum 'efficiency' is about 25 per cent. The energy is derived ultimately from the oxidation of foodstuffs.

(v) Little is yet known of the means by which 'excitation' induces chemical change and the latter mechanical effects. The existence (in some muscles) of optical phenomena running parallel with the thermal and mechanical changes, and the alterations of apparent viscosity during contraction, 
strongly suggest that the ultimate cause of the mechanical effects is a reversible rearrangement in a system of large protein molecules, on which $\mathrm{X}$-ray analysis, or the methods of surface chemistry, may ultimately throw light.

A not unimportant result of work on the heat production of muscle has been the enforced improvement of galvanometers and of thermopiles of the insulated type. This has had some interesting by-products; for example, (i) a sensitive method for measuring the slow resting heat production of a small object; (ii) a method of determining the vapour pressure depression of a small quantity of solution (down to $0 \cdot 1 \mathrm{mgm}$.) to an accuracy considerably better than 1 per cent; (iii) the possibility of further progress in studying stresses in engineering structures by means of their thermo-elastic properties; (iv) a method of increasing galvanometer sensitivity by the use of a photo-cell.

Most important, however, from the point of view of physiology, has been the work on the heat production of nerve. It was formerly believed that a nerve transmitted its messages without loss of energy, that the nerve impulse was in some sense analogous to a mechanical or electromagnetic wave in which all the energy involved is put into the system at the start. Unsuccessful attempts to measure the heat production of stimulated nerve supported this point of view. As a matter of fact, it is quite wrong: the heat is very small, but it is measurable and it obeys certain quite definite rules. In the transmission of a single impulse in medullated nerve, there is an 'initial' production of heat of $2 \times 10^{-8}$ to $8 \times 10^{-8}$ calorie per gram of nerve, and a recovery heat production of ten to thirty times that amount. The time-course of the latter, occupying 10-30 minutes (at $20^{\circ} \mathrm{C}$.), has been mapped out: the relation of the former to temperature, frequency of excitation and other factors has been determined.

The results have been confirmed by measurements of the oxygen consumption, and attempts have been made to determine the chemical changes underlying the transmission of the nervous impulse. These latter have not, as yet, had much success, owing to the extreme smallness of the quantities involved. It is probable that the first stage in nerve transmission is the building up at each point of a critical electrical potential, under the influence of the electric accompaniment of the impulse approaching from a distance. When the potential reaches its critical value at any point a state of instability of unknown nature results, the insulating properties of the nerve boundary change, and the potential difference normally existing at that boundary is now able to produce a current to neighbouring regions : these in turn are similarly activated and the wave is propagated. The nerve boundary then returns to its normal insulating state. The initial heat is presumably due to the chemical events associated with this cycle of membrane changes: as in muscle where, with 100,000 times as much heat, chemical changes are associated with contraction and relaxation. The recovery heat may be due either to an oxidative reversal of these chemical changes, or to the restoration of the ionic differences responsible for the resting potential difference across the nerve boundary.

For the moment, there is a lull in these measurements of heat in muscle and nerve: further progress is probably to be sought chiefly in other directions. Micro-chemical methods need to be greatly improved if the chemistry of nerve activity is to be understood; the physical chemistry of the active boundaries involved in nerve transmission requires an application of the new technique of surface chemistry; the electrical accompaniments of excitation and transmission need more accurate quantitative study. On the muscle side, owing to the greater magnitudes involved, the chemistry is not so difficult, and considerable light is being thrown, and a very complex system is being revealed, by biochemical methods. The newer methods, however, of optical and $\mathrm{X}$-ray analysis need to be more fully tried before one can say how far alteration in complex molecular systems will be found responsible for the mechanical accompaniments of activity. The effects of high hydrostatic pressure on the behaviour of muscle and nerve, and the changes of volume accompanying contraction, also may throw light on the mechanism.

These researches have been due to the cooperation of many. Langley started them on the suggestion of Fletcher and Hopkins's work. Bürker and Paschen, in respect of thermopiles and galvanometers, gave fundamental help. R. A. Peters, Weizsäcker, Parnas and Lovatt Evans collaborated before 1914. Hartree for many years bore the brunt of the experiments (and the arithmetic !) on the analysis of muscle heat into its constituent phases. Downing similarly contributed his skill in the construction of delicate instruments. The work of Meyerhof, of Embden, of Parnas, of the Eggletons, of Lundsgaard and of many others brought light from the chemical side. Fenn's fundamental researches on the relation between work and heat have been, and Gerard's on the subject of nerve metabolism must be, recorded. Azuma, Ernst Fischer, Gasser, Parkinson, Cattell, Furusawa, Wyman, Bronk, Bozler, Feng, Levin, Cowan, Bugnard, Rosenberg, each furnished a characteristic contribution. The list is not exhaustive. In spite of the 
present lull, at any stage in further progress reference may have to be made once more to the so-called 'myothermic' and 'neurothermic' methods, which have so great an advantage in their quickness and sensitivity, and in the fact that they can determine so fundamental a quantity as the total energy without any injury to the experimental object. The usefulness of these methods will probably not end with the jubilee of their revival.

\section{Therapeutic and other Applications of X-Rays and Gamma-Rays}

\section{By Dr. G. W. C. KaYe, o.B.E., National Physical Laboratory}

W ITHIN a period of fifteen years prior to the King's accession, Röntgen had discovered the X-rays and J. J. Thomson the electron, Becquerel had discovered radioactivity and the Curies had isolated radium. The new reign was to prove an era of X-ray and radium research no less fruitful than its predecessor. In particular, the X-ray crystal diffraction experiments of Laue in Germany in 1912, followed by those of the Braggs in England, opened up a new vista of research which has left its mark on physics, found diverse and important applications in industry and is beginning to acquire significance in the biological sciences. Röntgen, who had lived to see many of these developments, died in 1923 in his seventy-eighth year, poor in fortune, but consoled by the beneficent services which his discovery had rendered in the War.

The British Army entered the War relying entirely for its X-rays on induction coils and 'gas' tubes, both well-nigh obsolete now. About 1908, Snook developed the high-tension closed-core transformer with rotary-arm rectifier, and in 1913, Coolidge introduced the hot-cathode X-ray tube. Much development has since followed. The $\mathrm{X}$-ray tube of to-day is self-protected and shockproof, and is even claimed to be climate-proof! The complete equipment is earth shielded, permits precise control and is silent in operation. The high-tension transformer works in conjunction with one or other of the rectifying-valve and condenser circuits, which deliver either pulsating or constant high voltage to the X-ray tube. In routine medical radiology, these voltages range from $60 \mathrm{kv}$. to $200 \mathrm{kv}$., but $400 \mathrm{kv}$. and up to $1,000 \mathrm{kv}$. are now to be found in certain therapeutic centres.

In 1910, X-ray diagnosis was fairly well advanced, but radiation therapy was in its infancy. Protection for the worker was rudimentary or non-existent; and many injuries and deaths resulted. Not until 1921 were the British protection recommendations issued by a representative committee, and these, the first in any country, were used as a basis for international agreement four years later at Stockholm. These recommendations, which are revised triennially by an International Protection Commission, have been adopted throughout practically the whole civilised world. They have not only provided effective safeguards against the working dangers of $\mathrm{X}$-rays, but also have contributed, as no other factor has, to the better housing and general well-being of the X-ray and radium worker. Most radiology departments in 1910 were deplorably housed, whereas the light, roomy and well-ventilated departments of to-day are often a source of pride to hospitals.

Another vital step in the progress of radiation therapy was the adoption of the röntgen (r.) as the international unit of X-ray quantity or dose. The röntgen, which is an air-ionisation unit, was the subject of an international intercomparison in 1931 by the national laboratories of the United States, Germany and Great Britain, a very satisfactory measure of agreement resulting. It is probable that the röntgen will be adopted internationally for gamma radiation also, though further measurements on both gamma and high-voltage $\mathrm{X}$-radiation are required.

Radiography has found considerable application in the industries and arts, for example, in the examination for flaws and other defects in metal castings and forgings, welds and assembled components. Wooden aeroplane parts were examined radiographically in the War. Many millions of elinical thermometers under test have been expeditiously scrutinised at the National Physical Laboratory by the use of X-rays during the last ten years. Other industrial applications include the screening of electrical insulators and golf balls during manufacture. The National Gallery and the Courtauld Institute have recently installed $\mathrm{X}$-ray outfits for the examination of pictures and objects of art. Gamma-rays are resorted to in the radiography of metal specimens too thick for $\mathrm{X}$-rays to tackle.

It is, however, in the world of medicine and surgery that $\mathrm{X}$-rays and radium have been turned to account most outstandingly. X-ray diagnosis has improved in the last twenty-five years to an almost spectacular extent, and radiation therapy, though of more recent growth, has now established its claim to an important place in the treatment of malignant disease. The use of radium for eancer treatment was given impetus in Great 